\title{
Development of a total reflection X-ray fluorescence spectrometer for ultra-trace element analysis
}

\author{
M K TIWARI*, B GOWRISHANKAR, $V$ K RAGHUVANSHI, R V NANDEDKAR and \\ K J S SAWHNEY \\ Synchrotron Utilization Section, Centre for Advanced Technology, Indore 452 013, India
}

MS received 29 January 2002

\begin{abstract}
A simple and fairly inexpensive total reflection X-ray fluorescence (TXRF) spectrometer has been designed, constructed and realized. The spectrometer is capable of ultra-trace multielement analysis as well as performs surface characterization of thin films. The TXRF setup comprises of an X-ray generator, a slitcollimator arrangement, a monochromator/cutoff-stage, a sample reflector stage and an $X$-ray detection system. The glancing angle of incidence on the two reflectors is implemented using a sine-bar mechanism that enables precise angle adjustments. An energy dispersive detector and a GM counter are employed for measuring respectively the fluorescence intensities and the direct $\mathrm{X}$-ray beam intensity. A Cu-target $\mathrm{X}$-ray generator with its line focus window is used as an excitation source. The spectrometer is quite portable with its compact design and use of a peltier cooled solid state detector for energy dispersive detection. Alignment and characterization of the TXRF system has been performed and the minimum detection limits for various elements have been determined to be in the range of $100 \mathrm{pg}$ to $5 \mathrm{ng}$ even at low $\mathrm{X}$-ray generator powers of $30 \mathrm{kV}, 5 \mathrm{~mA}$. The capability of the TXRF system developed for thin film characterization is also demonstrated.
\end{abstract}

Keywords. XRF; TXRF; X-ray spectrometry; detection limits.

\section{Introduction}

Total reflection X-ray fluorescence (TXRF) spectroscopy is a modern technique for the determination of ultra-trace amount of elements in various kinds of samples. This technique is a variant of the energy dispersive X-ray fluorescence (EDXRF) analysis technique. In essence there are two major differences from conventional EDXRF: (i) the specimen is excited by the primary X-ray beam at a glancing angle less than the critical angle at which total external reflection occurs, and (ii) primary exciting radiation is incident on plane, polished surface, which serves either as a sample support or is itself the object to be examined.

As a consequence of above conditions, the effect of external total reflection exploits the high reflectivity on flat surfaces and low penetration depth of the primary X-ray beam. This allows the application of TXRF for ultratrace elemental analysis on the one hand and surface analysis on the other. In conventional EDXRF, the detection sensitivity is limited by the large Compton scattering of the primary X-ray beam from the sample bulk resulting in detection limits of the order of a few tens of ppm $(\mu \mathrm{g} / \mathrm{g})$ for most of the elements detectable by this

\footnotetext{
*Author for correspondence
}

technique. Because of the low penetration of the primary $\mathrm{X}$-ray beam in TXRF, the primary beam does not interact with the sample bulk thereby Compton scattering is drastically reduced resulting in improved sensitivities of sub-ppm levels. By TXRF technique, picogram amounts of elements ranging from $\mathrm{Cl}$ to $\mathrm{U}$ can be determined in aqueous residues in sample volumes of the order of $10 \mu \mathrm{l}$. The history of the development of TXRF instrumentation and technique has been summarized elsewhere (Yoneda and Horiuchi 1971; Aiginger and Wobrauschek 1974; Wobrauschek and Aiginger 1975; Knoth and Schwenke 1978) and there have been several time to time reviews which describe the principles of TXRF and various new design for its instrumentation to increase the capability of technique (Wobrauschek and Aiginger 1986; Yun and Bloch 1990; Wobrauschek et al 1991; Rieder et al 1993; Streli et al 1993; Noma and Iida 1994; Waldschlager 2000). Several experimental determinations reported in literature indicate that the detection capability of TXRF is in the range of nanogram to picogram. Present state of the arts detection limits are in the famtogram range, achieved by employing synchrotron radiation as an excitation source (Streli et al 1999; Awaji et al 2000).

The critical angle, below which total reflection occurs, is typically in milliradian region, depending on the energy of primary incident radiation. For example, for quartz or silicon substrate, the critical angle is less 
than $4.0 \mathrm{mrad}\left(\sim 0.21^{\circ}\right)$ for $\mathrm{Cu}-\mathrm{K}_{\alpha}$ energy. Most of the commercially available TXRF spectrometers use stepper motor driven goniometer stages to control such low angles of incidence for various reflector stages used in TXRF spectrometers. In addition to the precise control of the grazing angles, which is a key parameter to obtain total reflection, the height between the two reflector stages also need to be controlled precisely for properly defining the footprint position of X-ray beam on reflector surfaces. Moreover, to ensure the reproducible optical conditions, all optical components of TXRF spectrometer require good mechanical stability.

We present here a design for TXRF spectrometer that is relatively simple, fairly inexpensive and has good angle adjustment capability. This spectrometer can be used for trace element detection as well as for surface characterization. A compact peltier cooled solid state detector, which does not require liquid nitrogen for its operation, is used to measure the energy dispersive X-ray fluorescence spectra. This detector has rather moderate energy resolution, however, the limitation of poor resolution is offset to a large extent by employing sophisticated spectrum processing techniques based on non-linear least squares fitting (NL-LSF) procedures (Sawhney et al 2000). The initial characterization of the spectrometer has been performed using an indigenously built $\mathrm{Cu}$ target X-ray generator (Tiwari et al 1998) which at present operates only at low powers. Even at X-ray generator powers of only $\sim 150 \mathrm{~W}$, sub-nanogram detection limits have been achieved for most of the elements detected. To demonstrate the capabilities of the spectrometer, it has been used for trace element analysis of several samples as well as for the surface characterization of a few thin films.

\section{Spectrometer design}

For X-rays, the complex index of refraction $\bar{n}$ of all materials is $<1$ so that external total reflection takes place at an angle below the critical angle, $\theta_{c}$, given by

$$
\theta_{\mathrm{c}}(\mathrm{deg})=\frac{1 \cdot 65}{E_{\text {cut }}} \sqrt{\frac{Z \rho}{A}},
$$

where $Z$ is atomic number, $A$ the atomic weight and $\rho$ the density in $\mathrm{g} / \mathrm{cm}^{3}$. The angle, $\theta_{\mathrm{c}}$, is measured with respect to the sample surface and its value is very small for $\mathrm{X}$ ray energies-typically less than a degree. Equation (1) indicates that smaller the critical angle higher the X-ray energy is. Above the critical angle, the X-rays do not get reflected and are instead absorbed in the sample.

In order to make TXRF measurements for examination of thin layers and granular residues, the TXRF instrumentation should meet the following requirements (Klockenkamper 1997):

(A) The grazing angle of incidence capable of being varied between $0^{\circ}$ to $3^{\circ}$ in steps of $0.01^{\circ}$.

(B) Primary X-ray beam should be shaped like a strip of paper with a vertical height of $\sim 0.05 \mathrm{~mm}$ and horizontal width of $\sim 10 \mathrm{~mm}$.

(C) High energy part of the primary spectrum must be eliminated by a filter so that total reflection can occur at relatively larger grazing angles.

A schematic representation of TXRF spectrometer developed is shown in figure 1 . It essentially comprises of an X-ray generator, a slit-collimator arrangement, a monochromator stage (i.e. cutoff-reflector), a sample reflector

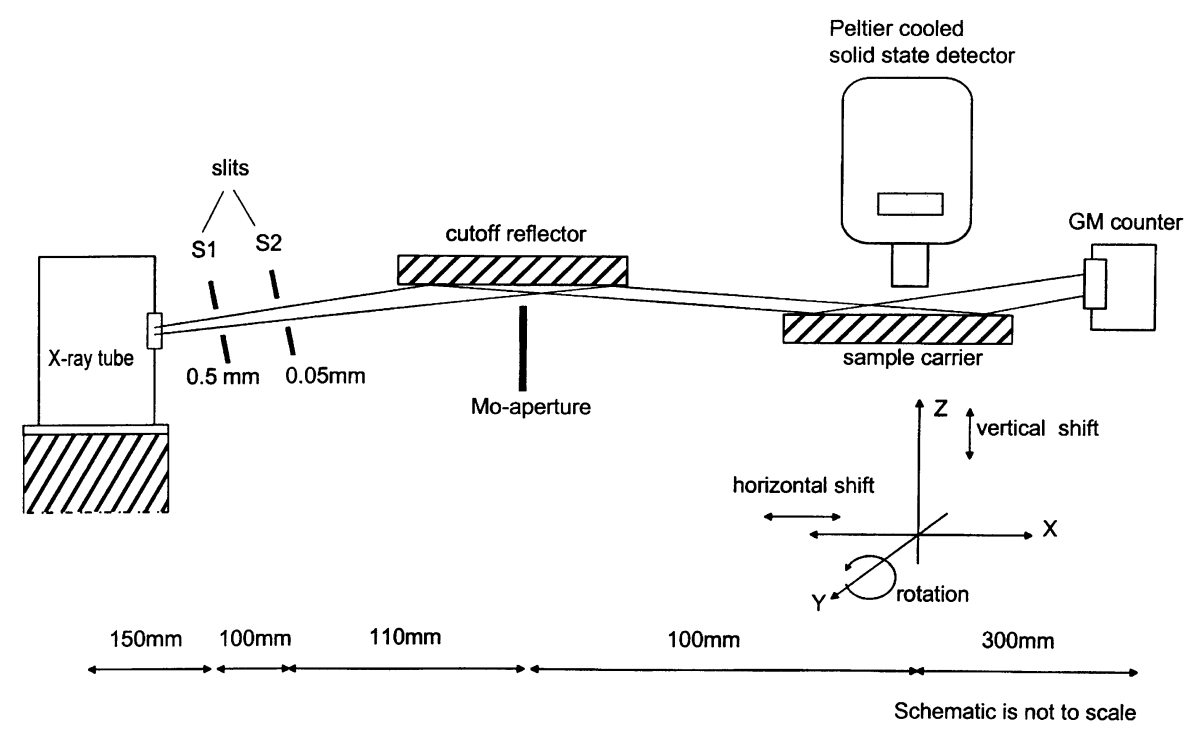

Figure 1. Schematic of the total reflection X-ray fluorescence (TXRF) setup. 
stage (i.e. sample carrier) and an X-ray detection system. The primary beam emitted by X-ray tube from its linefocus is collimated by the slit-collimator arrangement, which generates incident X-ray beam shaped like a strip of paper. A polished silicon crystal ( $\mathrm{Si}-111)$ of dimensions $30 \times 34 \mathrm{~mm}$, and $0.3 \mathrm{~mm}$ thickness, is mounted on the first reflector stage in downward looking direction to act as a cutoff reflector. The grazing angle on the cutoffreflector is set to such a value that it cuts off the higher energy part (high energy bremsstrahlung) of primary radiation thereby making the primary $\mathrm{X}$-ray beam quasimonochromatic. For example, for quartz or silicon, the relationship between the cutoff energy, $E_{\text {cut }}$, and critical angle, $\theta_{\mathrm{c}}$, can be simplified from (1) to

$$
E_{\text {cut }}[\mathrm{KeV}]=\frac{1 \cdot 73}{\theta_{\mathrm{c}}[\mathrm{deg}]} .
$$

This equation indicates that the X-rays with energies higher than $E_{\text {cut }}$ corresponding to the adjusted angle will not be reflected and will be absorbed in the reflector material. Suppression of high-energy bremsstrahlung radiation improves the signal-to-background ratio. The second reflector stage serves as sample carrier through which the fluorescence measurement of a sample material can be performed under external total reflection condition. A float glass is mounted as the sample carrier on this stage in the upward looking direction. A $2 \mathrm{~mm}$ thick Mo aperture is placed near the cutoff reflector stage to shield the sample-reflector from the direct primary X-ray beam.

The detection system for energy dispersive measurement consists of a peltier cooled solid state detector [Eurisys Mesures EPXR 10-300, $10 \mathrm{~mm}^{2}$ area], a spectroscopy amplifier AMP-6300 and a multi channel pulse height analyser installed in a personal computer. The solid state detector has an energy resolution of $240 \mathrm{eV}$ at $5.9 \mathrm{KeV}$ and operates without liquid nitrogen. The detector is placed very close and normal to the sample substrate at $\sim 4 \mathrm{~mm}$, so that a large solid angle is intercepted which thereby maximizes the counting efficiency. To monitor the intensity of the primary beam, a GM counter is placed in the specular reflection direction. The relative distances of slits, cutoff-reflector and sample carrier have been calculated assuming a $0.01^{\circ}$ source divergence. The total length of the spectrometer is $760 \mathrm{~mm}$.

\section{Mechanical description}

Most of the components of the TXRF spectrometer have been designed and constructed in-house. An isometric view of the TXRF attachment is shown in figure 2. The slit-collimator assembly $(1)^{1}$ comprises of a pair of precisely aligned Mo slits of dimensions $10 \times 0.5 \mathrm{~mm}\left(S_{1}\right)$ and $10 \times 0.05 \mathrm{~mm}\left(S_{2}\right)$, respectively and are separated

${ }^{1}$ Numerals in parenthesis refer to figure 2 . by $100 \mathrm{~mm}$. This slit-collimator assembly makes primary beam dimensions $10 \mathrm{~mm}$ wide and $0.05 \mathrm{~mm}$ thick with a beam divergence of $\sim 0.01^{\circ}$, which is small enough to obtain total external reflection. The vertical motion (Z-motion) as well as tilting motion ( $\phi$-motion) for whole slit-collimator assembly is provided by means of two micrometers. The angular tilt motion provided here can tilt X-ray beam around $\mathrm{X}$-axis in a controlled manner with a minimum step size of $\sim 0.01^{\circ}$ and is achieved by using sine-bar arrangement.

The two reflector stages, $(2)^{1}$ and $(3)^{1}$, have separate mechanical arrangements for rotation ( $\theta$-motion) as well as for the vertical shift (Z-motion). Two micrometers of least count $1 \mu \mathrm{m}$ are used for each reflector stage-one for vertical movement and the other for incident angle adjustment using sine-bar arrangement. Flexibility has been provided to mount various size sample carriers in the sample holder. Calibration of the $\theta$-motion has been done using $\mathrm{He}-\mathrm{Ne}$ laser and is found to be 3.2 $\pm 0.2 \mathrm{mdeg} / 10 \mu \mathrm{m}$ for each reflector stage. This calibration has been verified using X-rays (see below). The advantage of using a sine-bar arrangement for $\theta$-motions is that the backlash error is minimal as well as it is relatively inexpensive compared to the commercially available stepper motor driven goniometer stages. Moreover, variable $\mathrm{X}$-shift between the two reflectors is provided. Coarse $Y$-shift has also been provided for these reflector stages.

All the mechanical assemblies including the slit-collimator arrangement, the first reflector stage and the second reflector stage, are mounted on a rigid stainless steel base plate $(5)^{1}$ at appropriate relative distances. This base plate rests on a support (6) ${ }^{1}$ so that it can be adjusted by means of screws to an angle equal to take-off angle of the primary X-ray beam. The detector holder $(4)^{1}$, used for

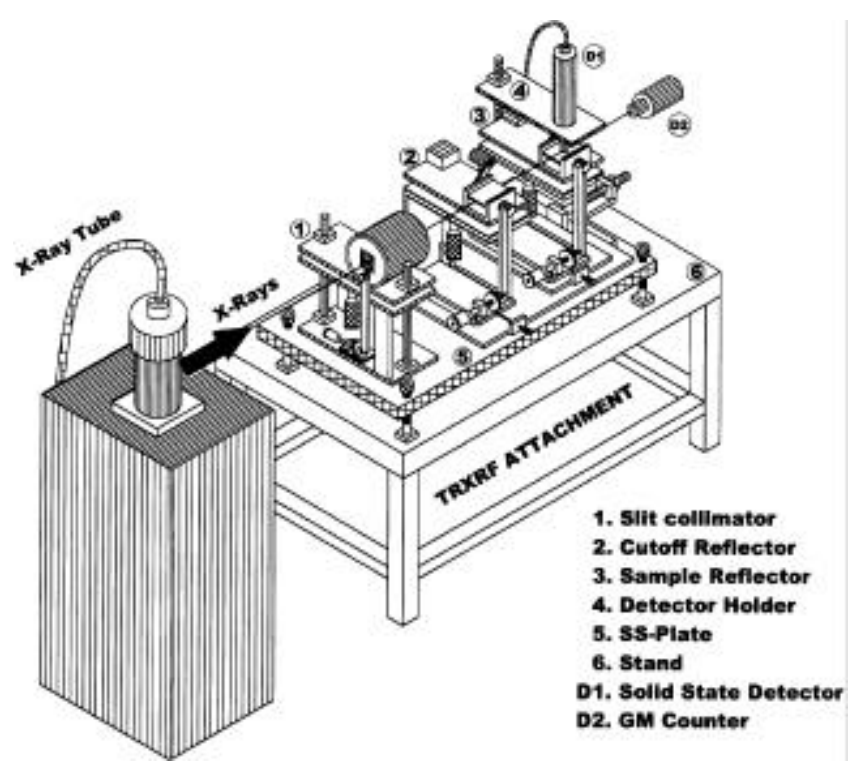

Figure 2. An isometric view of the TXRF attachment. 
mounting of peltier cooled detector, is made of perspex and is supported on a robust stand. This detector stand is mounted separately from TXRF base plate.

The TXRF module is connected directly to the window of X-ray generator. The spectrometer has provision for mounting of various types of optical elements such as cutoff-reflector, multilayer filter or natural crystal at the first reflector stage. Therefore, by choosing appropriate optical element, the energy bandwidth of primary radiation can be tailored to suit the excitation of a given sample. For example, for the analysis of granular residue materials, a cutoff-reflector would be a preferred choice and should be adjusted in such a way that both $\mathrm{K}_{\beta}$ and $\mathrm{K}_{\alpha}$ characteristic radiation of primary beam could be used for excitation. On the other hand, for thin film analysis, multilayer reflector is preferred as a spectrum modification unit at first reflector stage because a monochromatic incident beam would produce a better angle dependent intensity profile.

\section{Spectrometer characterization}

The TXRF spectrometer developed has been characterized in detail before putting it to actual use. To demon- strate the behaviour of low-pass filtering of cutoff reflector, the second reflector is removed from the X-ray beam path. The first reflector is adjusted in the primary beam path such that it intercepts half of the primary beam and at the same time is parallel to it. To measure the intensity of reflected X-rays, energy dispersive detector is placed in the specular reflection direction. For a given grazing angle, the first reflector allows all energies to be reflected up to a cutoff energy, $E_{\text {cut }}$, given by (2). Figure 3(a) shows the energy dispersive spectra acquired at various grazing incidence settings. This figure clearly shows that at decreasing grazing angles, X-ray energies from zero to progressively higher cutoff energies, $E_{\text {cut }}$, get reflected. The angular calibration of the sine-bar mechanism of this cutoff reflector, derived from the cutoff energies at various micrometer settings gives a value of $3.4 \pm 0.2 \mathrm{mdeg} / 10 \mu \mathrm{m}$. Similarly, one gets a calibration of $3 \cdot 2 \pm 0 \cdot 2 \mathrm{mdeg} / 10 \mu \mathrm{m}$ for the angular calibration of second reflector. These calibration values of angular motions of the two reflectors match quite well with the calibration done independently using laser light. After the angular calibration of the two reflectors, they are set at their respective positions. The first reflector is set to $\sim 0.21^{\circ}$ corresponding to the critical angle for $\mathrm{Cu}-\mathrm{K}_{\alpha}$ energy so that it allows to pass $\mathrm{X}$-ray energies up to

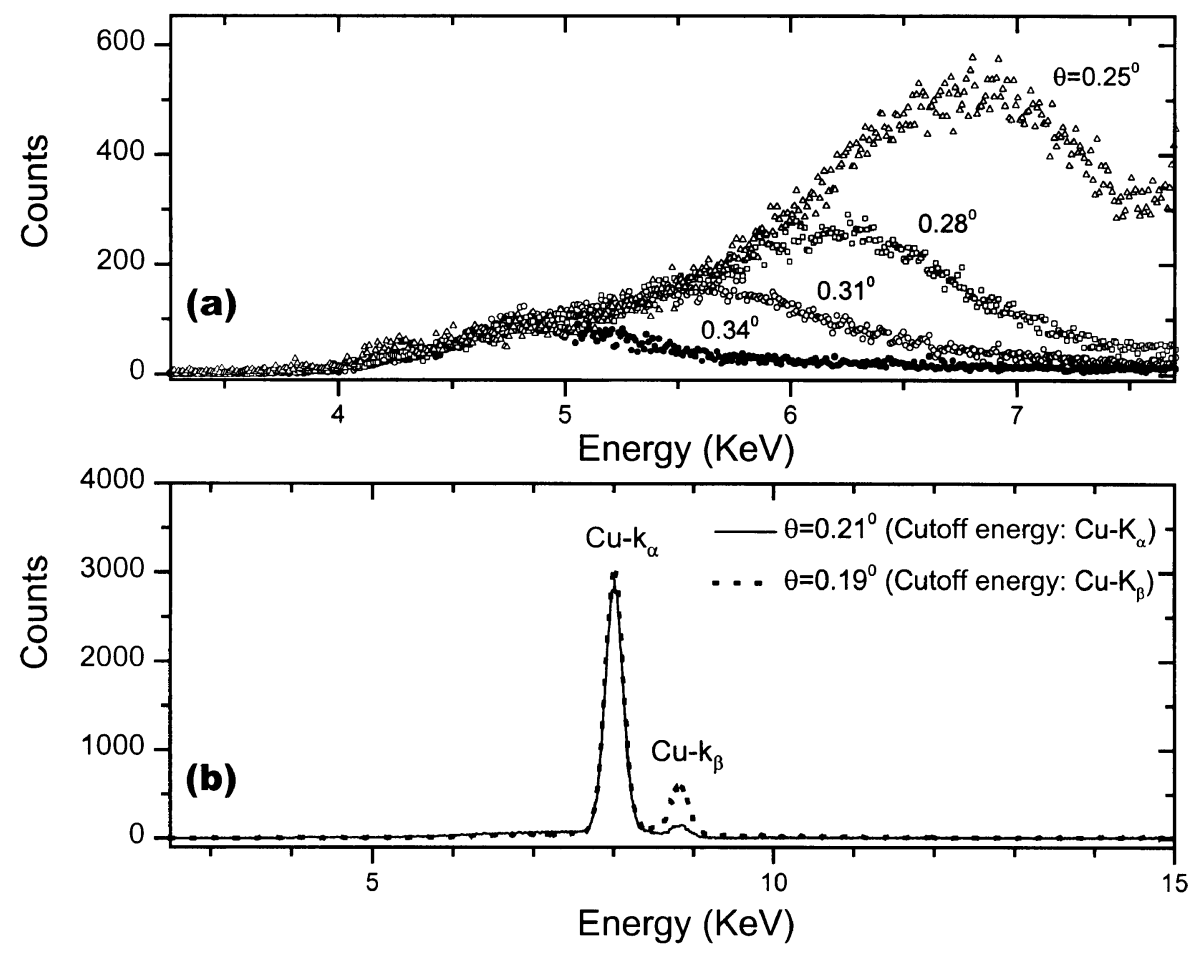

Figure 3. Characterization of first reflector with energy dispersive detector used in specular reflection mode: (a) reflected spectra at various incidence glancing angles. The figure shows that one gets higher cutoff energies for lower glancing angles as predicted by (2) and (b) energy dispersive spectra at glancing angles of $0 \cdot 21^{\circ}\left(0 \cdot 19^{\circ}\right)$ corresponding to cutoff energy of $\mathrm{Cu}-\mathrm{K}_{\alpha}\left(\mathrm{Cu}-\mathrm{K}_{\beta}\right)$. The $\mathrm{Cu}-\mathrm{K}_{\beta} / \mathrm{K}_{\alpha}$ intensity ratio reduces from 0.18 to 0.03 when glancing angle is changed from $0.19^{\circ}$ to $0 \cdot 21^{\circ}$ thereby indicating the good filtering action of the first cutoff reflector. 
$\mathrm{Cu}-\mathrm{K}_{\alpha}$ energy of primary beam thus making it quasimonochromatic. The sample reflector is adjusted such that it satisfies total external reflection condition for quasi-monoenergetic beam generated by cutoff-reflector. Figure 3(b) shows two spectra at two nearby angle settings. At one setting, X-ray energies up to $\mathrm{Cu}-\mathrm{K}_{\alpha}$ energy get reflected while at the other setting, energies up to $\mathrm{Cu}-\mathrm{K}_{\beta}$ get reflected. This shows the good control one has on the angle adjustment capability as $\mathrm{Cu}-\mathrm{K}_{\alpha}$ and $\mathrm{K}_{\beta}$ energies differ by about $900 \mathrm{eV}$ which corresponds to a change in critical angle of $\sim 0.02^{\circ}$ only.

To determine the detection limits (DLs) achieved with this spectrometer, various single element liquid standards of concentration $10 \mu \mathrm{g} / \mathrm{ml}$ were prepared from GR grade chemicals by mixing in de-mineralized water. $5 \mu \mathrm{l}$ volume of this solution is pipetted on to the sample carrier (float glass) using a micropipette and then dried. The residue left on the glass substrate is then analysed by TXRF spectrometer. All measurements were performed at $30 \mathrm{kV}, 5 \mathrm{~mA}$ tube power and at acquisition times of $1000 \mathrm{~s}$. The DLs determined for various elements are given in figure 4. The figure shows that the DL of $100 \mathrm{pg}-5 \mathrm{ng}$ is achieved for elements $\mathrm{Ca}$ to $\mathrm{U}$ even at the modest $\mathrm{X}$-ray tube powers employed. For the determination of minimum detection limit of uranium, $\mathrm{U}-\mathrm{M}_{\alpha, \beta}$ line intensity has been used.

\section{Results and discussion}

To demonstrate the effect of total reflection, a sample was analysed in two excitation geometries-normal excitation mode and total reflection mode. In normal excita-

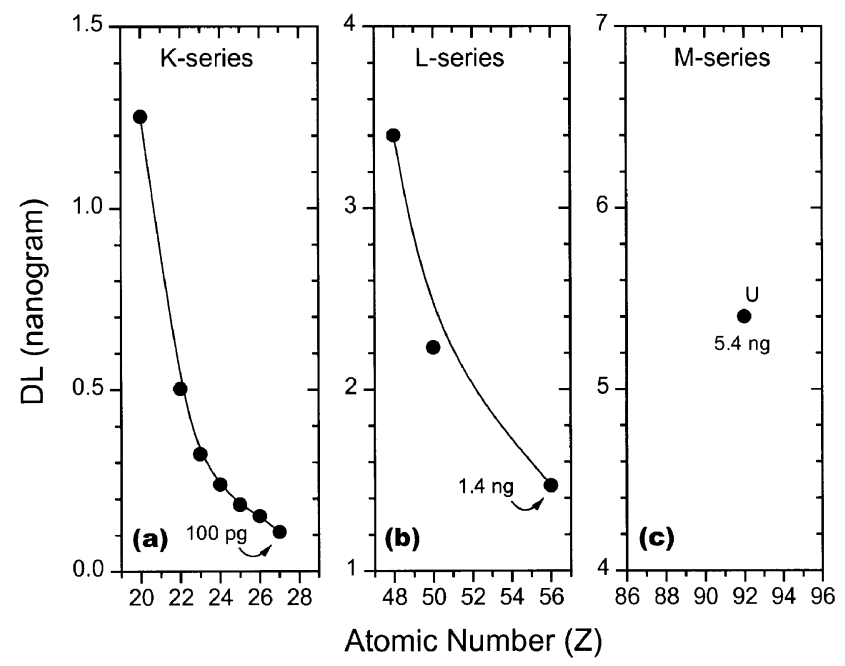

Figure 4. The detection limits determined by TXRF applied to residues of aqueous solutions at modest $\mathrm{X}$-ray tube powers. Excitation source: $\mathrm{Cu}$ X-ray tube, $30 \mathrm{kV}, 5 \mathrm{~mA}$. Analysis time: $1000 \mathrm{~s}$. Plots (a), (b) and (c), respectively show the DLs determined for $\mathrm{K}$-series, L-series and M-series fluorescence lines. tion mode, the grazing angle for sample carrier was kept at $\sim 0.25^{\circ}\left(\theta>\theta_{\mathrm{c}}\right.$, where $\theta_{\mathrm{c}}$ (float glass) $\left.\sim 0.21^{\circ}\right)$, whereas in total reflection mode it was kept at $0.18^{\circ}$ (i.e. below critical angle). From figure 5(a) it is clear that in normal excitation mode, X-ray beam penetrates deep into the glass material resulting in fluorescence of $\mathrm{Ca}-\mathrm{K}_{\alpha}$ from the glass substrate and a large scattering of primary radiation whereas in total reflection mode (figure 5(b)), primary X-ray beam excites only the specimen residue deposited on the carrier surface. It does not penetrate much into the glass material except a minimum depth (Schwenke and Knoth 1993). This reduces the background fluorescence $\left(\mathrm{Ca}-\mathrm{K}_{\alpha}\right)$ as well as produces substantial reduction (85 to $95 \%$ ) of primary scattering. At the same time, the fluorescence intensity of $\mathrm{Fe}$ is increased by $\sim 1.5$ times.

The angular dependence of total reflection X-ray fluorescence intensities can behave in various ways (Schwenke et al 1992; Kregsamer et al 1999). To evaluate the capability of our TXRF system in this respect, the angular dependence of fluorescence intensity of a $100 \AA$ thick Ti thin film deposited on a float glass substrate by electron beam evaporation system (Lodha et al 1996) has been examined. The thin film sample was mounted on the sample holder and the angle of incidence was varied in steps of $0 \cdot 01^{\circ}$. The fluorescence measurements have been taken for a period of $500 \mathrm{~s}$ at each angular position of the sample. The variation of $\mathrm{Ti}-\mathrm{K}_{\alpha}$ and $\mathrm{Ca}-\mathrm{K}_{\alpha}$ fluorescence intensities as functions of grazing angle of incidence is shown in figure 6 . The figure shows that the intensity of $\mathrm{Ti}-\mathrm{K}_{\alpha}$ increases with increase of incidence angle and reaches its maximum around critical angle of $\mathrm{Ti}$ for $\mathrm{Cu}-$ $\mathrm{K}_{\alpha}$ energy, which is found to be at $0.289^{\circ}$ and matches

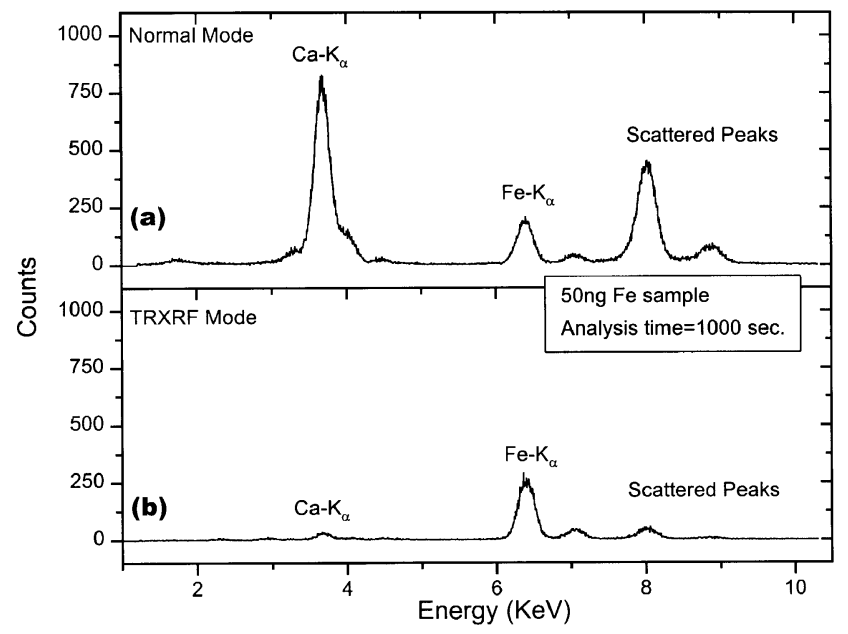

Figure 5. Energy dispersive X-ray fluorescence spectrum recorded from a $50 \mathrm{ng} \mathrm{Fe}$ sample in two excitation geometries $(\mathrm{Cu}$ tube, $30 \mathrm{kV}, 5 \mathrm{~mA}$, analysis time $1000 \mathrm{~s}$ ). (a) Normal excitation mode: glancing incidence angle sets above the critical angle and (b) TXRF mode: glancing incidence angle sets below the critical angle. 
very well with theoretical value of $0 \cdot 29^{\circ}$. The rise in fluorescence intensity is rather steep below the critical angle, as the specimen gets excited both by the primary beam and the reflected beam. Beyond the critical angle, the fluorescence intensity starts reducing as now there is no totally reflected beam to excite the specimen. At sufficient high incidence angles, the fluorescent intensity becomes more or less constant. On the other hand, $\mathrm{Ca}-\mathrm{K}_{\alpha}$ fluorescence intensity, which originates from the float glass substrate, is almost negligible below the critical angle of $\mathrm{Ti}$ because almost all primary intensity is reflected by Ti-thin film. It starts to increase after critical

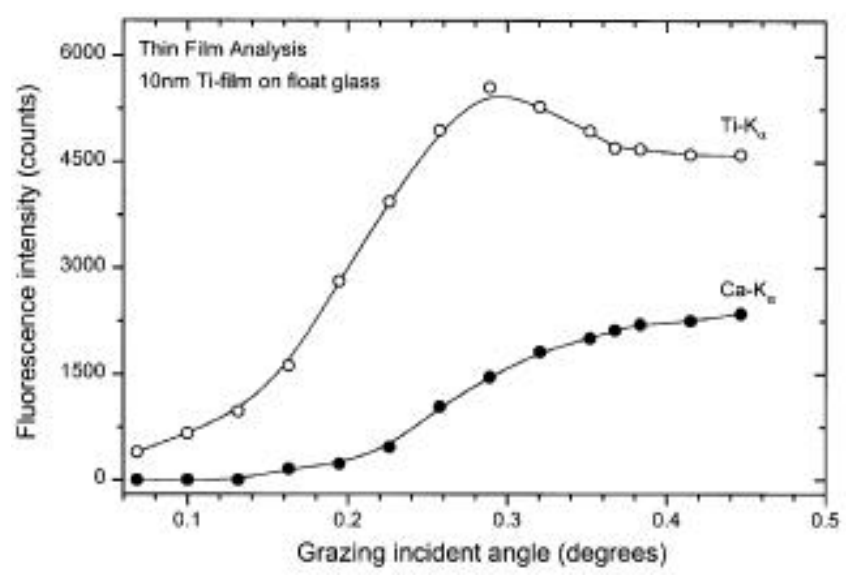

Figure 6. Angular dependence of fluorescence intensity examined in $10 \mathrm{~nm}$ thick Ti-film sample. Excitation condition: $\mathrm{Cu}$ tube, $30 \mathrm{kV}, 5 \mathrm{~mA}, 500 \mathrm{~s}$ for each angle setting.

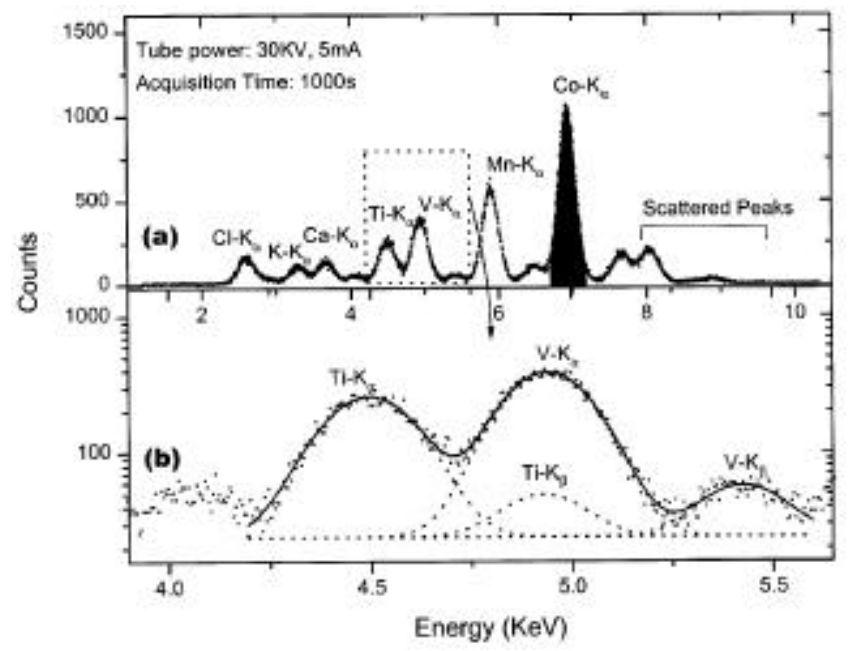

Figure 7. (a) Total reflection X-ray fluorescence spectrum obtained from a synthetic multielement sample comprising equal conc. $(10 \mu \mathrm{g} / \mathrm{ml})$ of $\mathrm{Ti}, \mathrm{V}, \mathrm{Mn}$ and $\mathrm{Co}$. Co is added as an internal standard. Excitation condition: $\mathrm{Cu}$ tube, $30 \mathrm{kV}, 5 \mathrm{~mA}$. Analysis time: $1000 \mathrm{~s}$ and (b) determination of net peak areas of the overlapped $\mathrm{Ti}$ and $\mathrm{V}$ peaks from nonlinear fitting procedure. angle of $\mathrm{Ti}$ and then becomes almost constant when the $\mathrm{Ca}-\mathrm{K}_{\alpha}$ fluorescence intensity from further depths in the substrate is not able to exit the sample surface. This example clearly shows the capability of the designed TXRF system for study of thin films and multilayer samples. Such studies are expected to yield information not only about the film thickness but also about the depth profile.

The quantitative analysis by TXRF spectrometry is relatively straight forward as the matrix effects, which are quite complex in the conventional EDXRF analysis, are minimal here because of very small sample mass getting used in this technique. Moreover, the normalization w.r.t. incident intensity, variation in the specimen residue area on the sample substrate, etc is readily done by using internal standards which are easily introduced since the sample residue are mostly generated from liquid samples. Figure 7(a) shows the TXRF spectra of a synthetic multielement sample comprising of equal weight fractions of elements, Ti, V, Mn and Co. Co is used as an internal standard. Owing to the poor energy resolution of the peltier cooled solid state detector used, the peaks of $\mathrm{Ti}$ and $\mathrm{V}$ strongly overlap. These peaks have been deconvoluted using NL-LSF procedures (figure 7(b)), and results thus obtained match quite well with the composition of the synthetic specimen.

Efforts are on to set up a $3 \mathrm{~kW}$ X-ray generator for use with the TXRF spectrometer developed, which would then increase the detection limits by at least an order of magnitude. Use of peltier cooled detector makes the TXRF system compact and facilitates easy handling compared to the oft used HPGe and $\mathrm{Si}(\mathrm{Li})$ detectors. The tradeoff is the poor energy resolution available from these detectors, which has been largely overcome by using spectrum deconvolution procedures based on NLLSF. However, for more demanding applications, provision has been made to mount a $\mathrm{Si}(\mathrm{Li})$ detector in the TXRF system developed. Use of a $\mathrm{Si}(\mathrm{Li})$ or HPGe detector will also improve the detection limits because they have generally larger active area $\left(\sim 30 \mathrm{~mm}^{2}\right)$ compared to the peltier cooled type detectors (in our case $10 \mathrm{~mm}^{2}$ ).

\section{Conclusions}

The design and development of a simple and fairly inexpensive total reflection X-ray fluorescence spectrometer is presented here. Such a simple TXRF spectrometer can be built in virtually any laboratory that has the basic XRF components (X-ray generator, energy dispersive detector). The absolute limits of detection achieved with peltier cooled detector at low tube power, using cutoff filter and float glass as a sample carrier ranges between $100 \mathrm{pg}$ and $5 \mathrm{ng}$. The instrument in its present condition has sufficient sensitivity for multielement analysis of a variety of geological and environmental samples. Further 
improvements are planned to be made by using a high power X-ray tube and a large area $\mathrm{Si}(\mathrm{Li})$ detector. Owing to the flexibilities built in the spectrometer, it can be used for multiple purposes including ultra-trace element analysis and surface characterization of thin films or Xray multilayer optical elements.

\section{Acknowledgements}

Authors thank G S Lodha for providing thin film sample. A K Singh is gratefully acknowledged for his help in sample preparation and data collection.

\section{References}

Aiginger H and Wobrauschek P 1974 Nucl. Instrum. Meth. 114 157

Awaji N et al 2000 Jap. J. Appl. Phys. Lett. 39 L1252

Knoth J and Schwenke H 1978 Fresenius Z. Anal. Chem. 291 200

Kregsamer P, Streli C, Wobrauschek P, Gatterbauer H, Pianetta P, Palmetshofer L and Brehm L Lora 1999 X-ray Spectrom. 28 292

Klockenkamper R 1997 in Total-reflection X-ray fluorescence analysis (New York: John Wiley and Sons, Inc.) Ch. 3 pp 87-105
Lodha G S, Nandedkar R V and Varma Adu 1996 Bull. Mater. Sci. 191109

Noma T and Iida A 1994 Rev. Sci. Instrum. 65837

Rieder R, Ladisich W, Wobrauschek P, Streli C and Kregsamer P 1993 Nucl. Instrum. Meth. A327 549

Sawhney K J S, Lodha G S, Kataria S K and Kulshreshtha S K 2000 X-ray Spectrum. 29173

Schwenke H and Knoth J 1993 in Handbook of X-ray spectrometry (eds) R E Van Grieken and A A Markowicz (New York: Marcel Dekker) Ch. 9, pp 456-458

Schwenke H, Gutschke R and Knoth J 1992 Adv. X-ray Anal. 35941

Streli C, Wobrauschek P, Unfried E and Aiginger H 1993 Nucl. Instrum. Meth. A334 425

Streli C et al 1999 Spectrochem. Acta B54 1433

Tiwari M K, Modi M H, Sawhney K J S, Nandedkar R V and Bapna S C 1998 Proc. 6th national seminar on X-ray spectroscopy and allied areas (eds) S K Joshi et al (New Delhi: Narosa) p. 126

Wobrauschek P and Aiginger H 1975 Anal. Chem. 47852

Wobrauschek P and Aiginger H 1986 Fresenius Z. Anal. Chem. 324865

Wobrauschek P, Kregsamer P, Streli C and Aiginger H 1991 Xray Spectrom. 2023

Waldschlager U 2000 Adv. X-ray Anal. 43418

Yoneda Y and Horiuchi T 1971 Rev. Sci. Instrum. 421069

Yun W and Bloch J 1990 J. Appl. Phys. 681421 